\title{
Carnets
}

Revue électronique d'études françaises de l'APEF

Deuxième série - 8 | 2016

Du Français en cause aux causes du français

\section{Réflexions autour des instructions officielles pour l'enseignement du français au Portugal : l'état des lieux}

Joaquim Guerra

\section{(2) OpenEdition}

Journals

\section{Édition électronique}

URL: http://journals.openedition.org/carnets/1883

DOI : 10.4000/carnets. 1883

ISSN : 1646-7698

Éditeur

APEF

Référence électronique

Joaquim Guerra, «Réflexions autour des instructions officielles pour l'enseignement du français au Portugal : l'état des lieux », Carnets [En ligne], Deuxième série - 8 | 2016, mis en ligne le 30 novembre 2016, consulté le 19 avril 2019. URL : http://journals.openedition.org/carnets/1883 ; DOI : 10.4000/ carnets. 1883

Ce document a été généré automatiquement le 19 avril 2019.

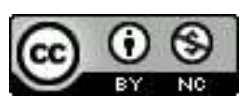

Carnets est mis à disposition selon les termes de la licence Creative Commons - Atribution - Pas d'utilisation commerciale 4.0 International. 


\title{
Réflexions autour des instructions officielles pour l'enseignement du français au Portugal : l'état des lieux
}

\author{
Joaquim Guerra
}

\section{Introduction}

Comme pour toutes autres disciplines enseignées au sein du système éducatif portugais, l'enseignement du français suit les normes des orientations officielles du Ministère de l'Éducation Nationale qui organisent les contenus (culturels/civilisationnels, fonctionnels et linguistiques) et fournissent des orientations méthodologiques plus ou moins obligatoires.

Or bien qu'au niveau de la recherche sur l'enseignement des langues vivantes étrangères d'autres approches méthodologiques soient actuellement défendues et malgré les successives recommandations et orientations du Conseil de l'Europe (e.g. le Cadre européen commun de référence pour les langues) et de l'Union Européenne, les programmes officiels portugais ne sont pas actualisés depuis les années 90 pour le cas du $3^{\text {ème }}$ cycle de l'enseignement basique (l'équivalent du collège français). C'est néanmoins le cycle qui accueille le plus grand nombre d'apprenants de Français.

3 Le but de cet article est de mener une réflexion au sujet des instructions officielles pour l'enseignement du Français au $3^{\text {ème }}$ cycle. Nous nous posons comme objectif de soulever, non de forme exhaustive, certaines des difficultés que le contexte décrit plus haut provoque. Nous organiserons cette réflexion par thèmes, ceux du moins que nous considérons pertinents, sachant dès le départ que ceux-ci ne cerneront pas tous les problèmes engendrés par la situation décrite. Nous finirons chacun d'entre eux avec quelques questions qui pourront inciter à de futurs développements d'analyses et de recherches. 


\section{L'organisation des différents niveaux d'apprentissage}

4 Le document actuel a pour principe qu'il existe deux différents groupes de niveaux d'apprentissage pour le $3^{\text {ème }}$ cycle de l'enseignement basique. Le premier, celui de continuation (Francês I), inclut les niveaux 3, 4 et 5 et le second, Francês II, les niveaux 1, 2 et 3 .

5 Or depuis que l'anglais est obligatoire au second cycle, dans lequel les étudiants ne peuvent choisir qu'une langue étrangère, le Francês I n'a aucun sens. En effet, seuls les étudiants qui au cycle précédent avaient choisi le français comme langue étrangère (deux ans d'études) pouvaient continuer leur apprentissage au troisième cycle et donc choisir le niveau 3 et ainsi de suite. Seuls les débutants pouvaient au $3^{\text {ème }}$ cycle commencer par le 1. D'ailleurs, les instructions officielles du Secondaire approuvées en 2001 (donc ultérieures) ne comportent plus les niveaux de 6 à 8, originellement fréquentés par les étudiants qui avaient commencé leurs études de français au second cycle.

De plus, il n'y a aucune référence aux échelles du cadre européen de référence (Conseil de l'Europe, 2001) puisque les instructions pour le $3^{\text {ème }}$ cycle sont antérieures à leur publication et que l'organisation par compétence, datant de 2001 (Abrantes, 2001), a été révoquée après la publication des nouvelles instructions officielles pour la plupart des disciplines scolaires après 2007.

7 Ainsi dit, personne ne sait à quel niveau de l'échelle du cadre correspond le Français 1, 2 ou 3 (les niveaux d'initiation de Francês I). Ce qui en fait pose problème étant donné que le cadre a pour principal objectif de faciliter la mobilité des étudiants et professeurs dans l'espace européen et pour cela il se constitue comme une référence pour la construction des programmes de langues nationaux, des manuels scolaires, de la formation des enseignants. En fait, le cadre (CECRL) a pour finalité :

D'offrir une base commune pour l'élaboration de programmes de langues vivantes, de référentiels, d'examens, de manuels, etc. en Europe. (...) En fournissant une base commune à des descriptions explicites d'objectifs, de contenus et de méthodes, le Cadre de référence améliorera la transparence des cours, des programmes et des qualifications, favorisant ainsi la coopération internationale dans le domaine des langues vivantes. Donner des critères objectifs pour décrire la compétence langagière facilitera la reconnaissance mutuelle des qualifications obtenues dans des contextes d'apprentissage divers et, en conséquence, ira dans le sens de la mobilité en Europe. (Conseil de l'Europe, $2000: 9$ )

8 Dans les divers pays, pour niveler les apprentissages effectués et ainsi faciliter la mobilité interne des européens, il fut également créé le portfolio européen des langues (Correia, 2001) et l'Europass.

9 Il faut ainsi poser une question pertinente : est-ce-que les élèves de $3^{\text {ème }}$ cycle seront en mesure de remplir le portfolio européen et de réellement certifier leurs compétences en Français Langue Étrangère (FLE) ? Nous rappelons que ce document est identique à toutes les langues de l'Union Européenne et sert de base, par exemple, aux étudiants qui désirent postuler pour des programmes Erasmus (ou qui en dehors du programme souhaitent étudier à l'étranger) ou aux adultes qui, pour diverses raisons, vont travailler dans un autre pays de l'espace européen et doivent donc remplir l'Europass. 


\section{Les questions méthodologiques}

10 Un choix méthodologique énoncé dans des programmes officiels nationaux n'est pas au départ uniquement le résultat de la recherche sur l'enseignement des langues ou sur les éléments qui constituent l'objet de l'apprentissage. Au fil des années, et surtout au Portugal où le système éducatif a souffert, en plus ou moins 10 ans, plusieurs reformulations d'instructions officielles dites nucléaires (comme le portugais ou les mathématiques) ce choix ne se justifie pas uniquement à partir des résultats des examens nationaux des étudiants ou des champs instables de la recherche sur les méthodologies car celles-ci sont à la fois théorie, pratique et théorie de la pratique et se fondent sur de nombreux champs disciplinaires (cf. Beacco, 2007). Les options méthodologiques s'appuient aussi sur des questions idéologiques, géopolitiques, d'harmonisation méthodologique entre langues enseignées, etc. d'un gouvernement qui gère le système éducatif et ses dispositifs.

Selon Beacco (2007 : 16-17),

(...) les méthodologies d'enseignement sont à considérer comme des ensembles solidaires de principes et d'activités d'enseignement, organisés en stratégies, fondées en théorie (c'est-à-dire qui s'appuient sur des concepts ou des connaissances élaborés au sein d'autres disciplines impliquées dans l'enseignement des langues) et/ou par la pratique (pas son efficacité, par exemple) et dont la finalité est d'accompagner les apprentissages.

La méthodologie d'enseignement doit accompagner l'apprentissage, ce qui veut dire «aider l'apprenant à 'mettre de l'ordre' dans ses observations ou à confronter sa perception intuitive des fonctionnements réguliers de la langue cible » (Beacco, $2007: 17$ ).

Depuis 2001, l'enseignement des langues au Portugal se fonde sur l'entrée par les tâches, une approche par compétences langagières (Abrantes, 2001). Cette approche, née dans l'enseignement professionnel, s'est rapidement répandue surtout après la publication du CECRL et sa perspective actionnelle «en ce qu'elle considère avant tout l'usager et l'apprenant d'une langue comme des acteurs sociaux ayant à accomplir des tâches (qui ne sont pas seulement langagières) dans des circonstances et un environnement donnés, à l'intérieur d'un domaine d'action particulier » (Conseil de l'Europe, $2001: 15$ ).

Le CECRL caractérise l'usage et l'apprentissage d'une langue de cette forme :

L'usage d'une langue, y compris son apprentissage, comprend les actions accomplies par des gens qui, comme individus et comme acteurs sociaux, développent un ensemble de compétences générales et, notamment une compétence à communiquer langagièrement. Ils mettent en œuvre les compétences dont ils disposent dans des contextes et des conditions variés et en se pliant à différentes contraintes afin de réaliser des activités langagières permettant de traiter (en réception et en production) des textes portant sur des thèmes à l'intérieur de domaines particuliers, en mobilisant les stratégies qui paraissent le mieux convenir à l'accomplissement des tâches à effectuer. Le contrôle de ces activités par les interlocuteurs conduit au renforcement ou à la modification des compétences. (Conseil de l'Europe, $2001: 15$ )

Donc, et encore selon Beacco (2007:11),

on y postule que la finalité principale de l'éducation est de former les apprenants à mobiliser leurs savoirs à bon escient et à les relier aux situations dans lesquelles ceux-ci permettent d'agir: 'l'actualisation de ce que l'on sait dans un contexte 
singulier (...) est révélatrice du passage à la compétence. Celle-ci se réalise dans l'action.' la majeure partie des programmes de langues étrangères défend l'entrée par les tâches ou méthodologie par les tâches (cf. Candlin, 1987; Nunan, 1989 et 2004; ou même les programmes de Français pour l'enseignement secondaire : Santos, 2001). concrétisation des abordages communicatifs de l'enseignement des langues dont le programme du $3^{\text {ème }}$ cycle s'inscrit. Nous n'irons pas présenter l'approche communicative. De nombreuses études ont été publiées sur ce thème et ses grandes lignes sont largement diffusées. Nous retrouvons dans les instructions officielles de 1993 les grands principes qui lui sont associés, comme par exemple :

- La centration sur l'apprenant et ses besoins langagiers ;

- La progression qui respecte les rythmes d'apprentissage individuels ;

- L'utilisation d'une grammaire de communication, fonctionnelle (organisation de contenus à partir d'actes de paroles) ;

- L'organisation des contenus à partir de domaines de référence thématiques ;

- L'utilisation de documents dits "authentiques», pour que l'authenticité discursive soit considérée normale.

Il est néanmoins difficile de comprendre comment les contenus grammaticaux convergent avec les domaines de référence puisque la liste est très étendue pour chaque année d'apprentissage, particulièrement pour le Francês I, réduisant, selon nous, le cours de FLE à un limitatif cours de grammaire. D'ailleurs, il est contradictoire de défendre une grammaire de communication, sous la forme d'analyse du discours (de réception et de production comme il est mentionné pages 20 à 21 et 51 à 56), et établir une liste de contenus grammaticaux si étendue. Les concepteurs des programmes courent le risque de provoquer l'effet inverse et que les enseignants pratiquent de la grammaire formelle (avec ses règles et exceptions), sous des méthodologies plus traditionnelles, bien plus rassurantes quand nous devons accomplir tout un programme annuel en soi si chargé.

Par ailleurs, l'emploi de catégories compréhensibles par les apprenants "parce que relevant de leur expérience communicative" (Beacco, 2007: 64) justifie, dans les abordages communicatifs, l'utilisation des actes de parole, théorisés dans les années 60 par Austin (1962) et Searle (1969) sous d'autres courants méthodologiques, plus structuralistes. Il serait donc, selon Widdowson (1998, apud Beacco 2007: 56-57) de " passer d'une conception de l'apprentissage des langues comme skill (c'est-à-dire comme intériorisation mécanique de la connaissance d'un code) à une autre conception de ces apprentissages, comme connaissance des conventions de leur emploi en contexte(s) (...) » mais qui ne facilite pas la systématisation du code verbal de base.

Finalement, il est important de souligner que malgré les différentes considérations méthodologiques au sujet des quatre domaines de communication (à ne pas confondre avec les domaines de référence que l'on retrouve aussi dans les instructions officielles) écouter, parler, lire, écrire - celles-ci sont générales, de difficile compréhension (particulièrement en ce qui concerne la grammaire ou de comment les développer dans les cours en interaction avec les contenus). Tavares, Valente \& Roldão (1997) disaient que «se limitam a reconhecer os princípios pedagógicos gerais (...), pelo que se torna muito difícil que as práticas dos professores com menos formação reflitam a orientação metodológica proposta » (p. 63). 
21 Il faut aussi souligner que le Currículo nacional do E.B. (Abrantes, 2001), entretemps supprimé, établissait en quelque sorte une actualisation méthodologique et organisationnelle des instructions officielles de 1993. En effet, et dû au dialogue constant établit par les auteurs du Currículo nacional do E.B. (Abrantes, 2001) et le CECRL, il déplaçait les programmes de la perspective communicative adoptée dans ceux-ci vers une perspective actionnelle, organisant l'enseignement $d u$ français au sein de tout l'enseignement basique (premier, second et troisième cycle).

Par conséquent, qui suggère actuellement quelle(s) méthodologie(s) doit/doivent être utilisée(s) dans l'enseignement du Français? Les enseignants? Les manuels scolaires? Suivent-elles toujours les choix méthodologiques du Currículo nacional do E.B. (Abrantes, 2001) qui, comme nous l'avons dit plus haut, a été révoqué?

\section{Les contenus et leur organisation}

23 Au cours du chapitre précédent, Nous avons déjà évoqué les contenus, notamment quant à leur organisation et leur relation avec la méthodologie élue pour l'enseignement du FLE, pour l'organisation des séquences didactiques. Nous rappelons qu'il n'est en aucun cas question de compétences, mais de contenus fonctionnels organisés autour de thèmes de communications.

24 Ainsi, les instructions officielles organisent les contenus autour de domaines de références dans lesquelles nous trouvons les actes de paroles et les champs thématiques (listes lexicales). Les contenus grammaticaux sont énumérés dans des listes séparées et seront mis en relation avec les différents domaines, à notre avis, selon les actes de paroles énoncés et d'après la lecture des enseignants ou des concepteurs de manuels scolaires. D'ailleurs, il est expliqué que la grammaire ne peut être enseignée que d'une forme contextualisée puisqu'il s'agit d'une grammaire au service de la communication (AAVV, 1994 : 21). Cependant nous pouvons trouver antinomique le fait que l'on présente « de forma sistematizada conteúdos que se prendem com a competência linguística, apesar de [os autores] colocarem a ênfase na abordagem comunicativa» (Tavares, Valente \& Roldão, 1997 : 66).

Bien que les domaines de références puissent être considérés comme méthodologiquement actuels car nous y retrouvons d'une manière générale les domaines de communication du CECRL (public, professionnel, éducationnel et personnel), il serait temps d'actualiser les sous-thèmes (il n'y a plus de P.T.T en France, par exemple) et de les organiser de manière à suivre les directives du CECRL, communes aux autres langues étrangères enseignées au $3^{\text {ème }}$ cycle.

Il faut aussi ajouter qu'il n'y a pas d'articulation verticale entre les Instructions du $3^{\text {ème }}$ cycle et celles du secondaire. En effet, Tavares (2001) affirme qu'une lecture des programmes du secondaire nous montre que «esta articulação parece-me não se ter verificado, desconhecendo os autores dos programas do secundário os programas do ensino básico » (p. 68).

Il existe déjà des publications qui se constituent comme des référentiels pour le FLE que les concepteurs de programmes peuvent utiliser pour une nouvelle organisation des contenus et compétences, à savoir, par exemple, Beacco \& Porquier (e.g. 2007), Chauvet \& Normand (2008), ou Beacco et al (2010). 
Ce qui nous mène à nous demander: Comment s'organisent les contenus (les compétences ?) pour chaque année du $3^{\text {ème }}$ cycle ? Suivent-ils toujours les programmes actuels? Si non, qui les a adaptés? Se fondent-ils sur les recommandations du Currículo nacional do EB (Abrantes, 2001)?

\section{En guise de conclusion}

Contrairement au niveau secondaire (cf. Tavares, 2001), nous constatons que pour le $3^{\text {ème }}$ cycle il n'y a aucune convergence entre les instructions officielles et les différents documents européens ou, au niveau national, avec les autres programmes de langues vivantes enseignées. De plus, comme il a été souligné, le document qui d'une certaine manière établissait des ponts entre les instructions officielles et le CECRL, le Currículo nacional do EB (Abrantes, 2001) n'est plus en vigueur depuis 2007.

Finalement, il nous faudrait, pour avoir une vue plus étendue et éclaircie, une étude de fond qui inclut les manuels scolaires et les pratiques des enseignants pour un bilan sur l'enseignement du FLE au $3^{\text {ème }}$ cycle ou à l'école d'une manière générale.

31 Néanmoins, il serait temps que le Ministère de l'Éducation reformule les instructions officielles. Et étant donné que les équipes du Ministère sont actuellement en train de reformuler les programmes nationaux pour le portugais et l'anglais, pourquoi ne pas en profiter pour revoir ceux de français?

\section{BIBLIOGRAPHIE}

AAVV (1994). Programas de Francês. Plano de organização do ensino-aprendizagem. Ensino Básico (3ํciclo) . Lisboa : ME/DGEBS.

ABRANTES, Paulo (2001). Currículo essencial do ensino básico. Competências essenciais. Lisboa : ME/DEB. AUSTIN, John L. (1962). How to do things with words. Harvard: Harvard University Press. BEACCO, Jean-Claude (2007). L'Approche par compétences dans l'enseignement des langues. Paris : Les Éditions Didier.

BEACCO, Jean-Claude \& PORQUIER, Rémy (2007). Niveau A1 pour le Français : utilisateur/apprenant élémentaire. Paris : Les Éditions Didier.

BEACCO, Jean-Claude et al. (2010). Guide pour le développement et la mise en cuvre de curriculums pour éducation plurilingue et interculturelle (disponible en ligne http://www.coe.int/t/dg4/linguistic/ ListDocs_Geneva2010.asp\#P58_2278).

CANDLIN, Christopher (1987). Toward task-based learning. In C. CANDLIN \& D. MURPHY (Eds.), Language learning tasks (pp. 5-22). Englewood Cliffs: Prentice-Hall.

CANDLIN, Christopher, \& MURPHY, Dermot (Eds.). (1987). Language learning tasks. Englewood Cliffs : Prentice-Hall International. 
CHAUVET, Aude \& NORMAND, Isabelle (2008). Référentiel de programmes pour l'Alliance Française élaboré à partir du cadre européen commun (A1, A2, B1, B2, C1, C2). Paris : CLE Internationale.

CONSEIL DE L'EUROPE (2001). Cadre européen commun de référence pour les langues : apprendre, enseigner, évaluer. Paris : Les Éditions Didier.

CORREIA, Helena et al. (2001). Portefólio europeu das línguas. Educação Básica (10-15 anos). Lisboa :

Ministério da Educação.

NUnAN, David (1989). Designing tasks for the communicative classroom. Cambridge: Cambridge

University Press.

NUNAN, David (2004). Task-based language teaching. Cambridge : Cambridge University Press.

SANTOS, Zélia S. (Coord.) (2001). Programa de Francês. Níveis de continuação e de iniciação $\left(10^{\circ}, 11^{\circ}\right.$ e $12^{\circ}$

anos). Cursos gerais. Formação geral e específica. Lisboa : Ministério da Educação.

SEARLE, John (1969). Speech Acts. Cambridge: CUP.

TAVARES, Clara F. (2001). Dos textos do Conselho da Europa aos programas de Francês do ensino secundário. As línguas na Europa : intercompreensão, desafios e ações. Intercompreensão. Revista de Didática das Línguas, 9, pp. 65-89.

tavares, Clara F., VAlente, Maria T. \& Roldão, Maria do Céu (1997). Dimensões formativas de disciplinas do ensino básico : língua estrangeira. Lisboa : Instituto de Inovação Educacional.

\section{NOTES}

1. L'approche par compétences a été transposée méthodologiquement à travers l'entrée par les tâches dans les programmes d'Anglais ou d'Espagnol qui ont été revus plus récemment. Cette approche actionnelle, présente dans le Currículo Nacional do EB (Abrantes, 2001) n'est néanmoins plus en vigueur pour le Français bien que nous croyons que les éditeurs ou les professeurs continuent, d'une certaine manière, et selon leurs perceptions, sur la même car la formation a été assez rare, à la transposée dans les manuels scolaires et en salle de classe.

\section{RÉSUMÉS}

Cet article a comme objet de réflexion les instructions officielles actuelles pour l'enseignement du Français (comme langue étrangère) au Portugal du $3^{\text {ème }}$ cycle de l'enseignement basique (l'équivalent au collège français). En effet, depuis sa sortie en 1993, il n'a souffert aucune reformulation/substitution comme pour d'autres disciplines du collège, notamment de langues. Nous nous fixons donc comme objectif de réfléchir sur cette situation qui nous semble fortement contradictoire.

This paper aims to reflect about the syllabi for the French language in the third cycle of the Portuguese basic education. In fact, since their publication in 1993, they never were substitute or reformulated like other syllabi from other school subjects although it represents the cycle that more students choose this language to study. 
INDEX

Mots-clés : instructions officielles, enseignement du FLE, troisième cycle

Keywords : syllabi, French teaching, third cycle

\section{AUTEUR}

JOAQUIM GUERRA

Universidade do Algarve

jguerra[at]ualg.pt 